\title{
Searches for New Physics in Events with Multiple Leptons with the ATLAS Detector
}

\section{David Yu*}

On behalf of the ATLAS Collaboration

Lawrence Berkeley National Laboratory and UC Berkeley

E-mail: dryuelbl.gov

\begin{abstract}
Events containing several leptons are useful probes of new phenomena due to the low background from Standard Model processes. We look for anomalous production of events with three or more leptons, and search for excited leptons, heavy leptons and heavy neutrinos. The searches use data recorded in 2011 at $\sqrt{s}=7 \mathrm{TeV}$ and in 2012 at $\sqrt{s}=8 \mathrm{TeV}$ by the ATLAS experiment at the LHC.
\end{abstract}

XXI International Workshop on Deep-Inelastic Scattering and Related Subject -DIS2013, 22-26 April 2013

Marseilles, France

\footnotetext{
* Speaker.
} 


\section{Introduction}

Final states containing multiple leptons are an important tool in searches for new phenomena beyond the Standard Model at the LHC. Searches using multilepton final states are motivated by several models of phenomena beyond the Standard Model, and benefit from low Standard Model backgrounds, particularly when requiring the leptons to be energetic, isolated, and consistent with originating promptly from the primary interaction vertex. The backgrounds are divided into two categories: irreducible backgrounds, where several energetic, isolated, and prompt leptons are produced through Standard Model processes such as $W Z$ and $Z Z$ production, and non-prompt and fake backgrounds, where leptons arise from semileptonic heavy flavor decays, misidentified jets, or asymmetric photon conversions.

These proceedings describe three such searches with the ATLAS detector [1]. The first search, motivated by the type III neutrino seesaw mechanism, looks for pair production of heavy fermions decaying to a four-lepton final state, including two leptons from an on-shell $Z$ boson. The second search looks for excited leptons in a final state with two leptons and a photon. The third search is a model-independent analysis of trilepton final states in many signal regions, intended to provide broad sensitivity to new phenomena with multilepton signatures.

\section{Type III Seesaw Model Heavy Fermions}

The type III neutrino seesaw mechanism postulates the addition of triplets of heavy fermions to the Standard Model, which generate the dimension-5 operator of the usual seesaw mechanism:

$$
N=\left(\begin{array}{cc}
\frac{1}{\sqrt{2}} N^{0} & N^{+} \\
N^{-} & -\frac{1}{\sqrt{2}} N^{0}
\end{array}\right)
$$

The model bears special interest for the LHC for several reasons. First, since $N$ transforms in the adjoint representation of $S U(2)$, it couples to the electroweak gauge bosons, and the production rate is not suppressed by Yukawa couplings. Second, the generated neutrino mass is of form $m_{v} \sim \frac{Y_{N}^{2}}{M_{N}}$, hence the mass of the new heavy states may be within reach of the LHC, of order $m_{N} \sim 100 \mathrm{GeV}$, if the Yukawa couplings are small, or if an inverse seesaw mechanism is invoked [2]. Finally, the heavy fermions mix with the Standard Model leptons, enabling decay to leptonic final states.

The analysis, using $5.8 \mathrm{fb}^{-1}$ of $p p$ collisions at $\sqrt{s}=8 \mathrm{TeV}$, searches for pair production of a charged and a neutral heavy fermion, where the charged fermion decays as $N^{ \pm} \rightarrow Z l^{ \pm} \rightarrow l^{+} l^{-} l^{ \pm}$, and the neutral fermion decays as $N^{0} \rightarrow W^{ \pm} l^{\mp}$, creating at least four leptons in the final state [3].

\subsection{Event Selection}

Events are required to have at least four electrons or muons. Hadronically decaying tau leptons are not considered. The leading lepton must have $p_{T}>25 \mathrm{GeV}$, while the remaining three leptons must have $p_{T}>10 \mathrm{GeV}$. The leptons are required to pass tight particle identification criteria [4, 5]. Electrons are required to lie within $|\eta|<2.47$, excluding the transition region between the barrel and endcap calorimeters, $1.37<|\eta|<1.52$. Muons are required to lie within $|\eta|<2.5$. To suppress the non-prompt and fake backgrounds, the non-triggered leptons are required to be isolated, with isolation parameters of 0.15 for muons and 0.16 for electrons, and to have a track 
consistent with originating from the primary interaction vertex, with transverse impact parameter significance $\frac{d_{0}}{\sigma_{d_{0}}}<3$.

$Z$ candidates are reconstructed from a same-flavor, opposite-sign lepton pair with invariant mass within $10 \mathrm{GeV}$ of the $Z$ mass. The third lepton candidate is taken to be the nearest in azimuthal angle to the reconstructed $Z$ momentum. The fourth lepton is chosen as the lepton with highest $p_{T}$ remaining.

\subsection{Background and Results}

Irreducible backgrounds from Standard Model ZZ production are suppressed by vetoing events with a second $Z$ candidate. The requirement of a fourth lepton is effective at suppressing backgrounds from $W Z$ production and $Z+$ jets where a jet is misidentified as a lepton. After these criteria are applied, the dominant background is $Z Z^{*}$, with smaller contributions from $Z Z Z^{*}, Z W W^{*}$, $t \bar{t}+W / Z$, and $Z+$ jets. All backgrounds are taken from simulation; in the case of $Z+$ jets, the background shape is taken from a sample where the impact parameter requirement is reversed on the third lepton, the isolation criterion is loosened, and only three leptons are required in the event.

The reconstructed $m_{N^{ \pm}}$distribution is shown in figure 1a, showing no significant excesses above the background predictions. At $m_{N^{ \pm}}=420 \mathrm{GeV}$, the probability to have equal to or more than the observed number of events with a background only hypothesis, $p_{0}$, is 0.20 . The corresponding upper limits at $95 \% \mathrm{CL}$ on $\mathscr{B}_{\text {ZWll }}=\mathscr{B}\left(N^{ \pm} \rightarrow Z l^{ \pm}\right) \times \mathscr{B}\left(N^{0} \rightarrow W^{ \pm} l^{\mp}\right)$ versus $m_{N^{ \pm}}$are shown in figure $1 \mathrm{~b}$. The mixing angles for electrons and muons are taken to be the maximal allowed, $\left|V_{e}\right|=5.5 \times 10^{-2}$ and $\left|V_{\mu}\right|=6.3 \times 10^{-2}$ [6], with $\left|V_{\tau}\right|=0$. In the case $\mathscr{B}_{Z W l l}=1$, a heavy charged fermion with $m_{N^{ \pm}}<350 \mathrm{GeV}$ is excluded ( $350 \mathrm{GeV}$ expected). In the case of a nominal massdependent branching fraction [7], $m_{N^{ \pm}}<245 \mathrm{GeV}$ is excluded (243 GeV expected).

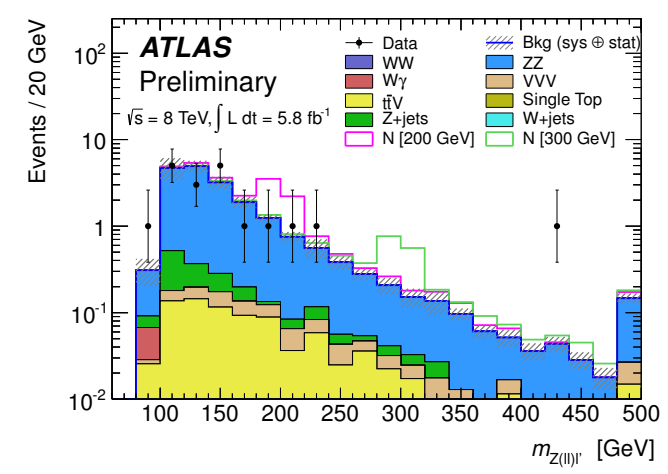

(a)

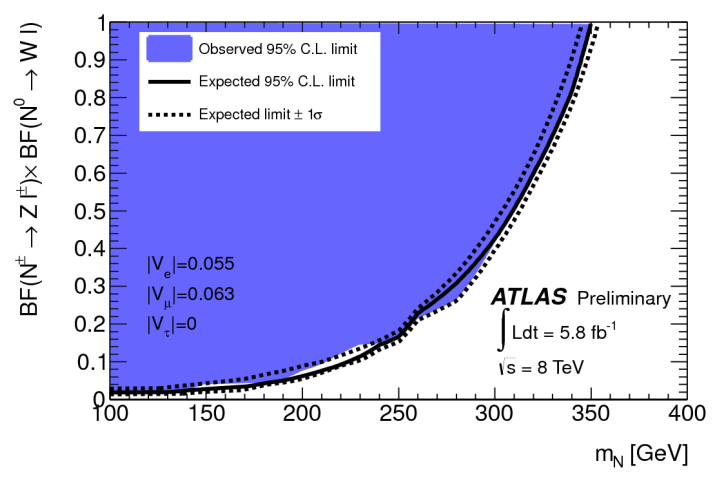

(b)

Figure 1: Left: Invariant mass distribution of the $N^{ \pm}$candidates in the signal region, for data and the expected total background. The rightmost bin includes overflow events. Right: The expected (dashed line) and observed (solid line) upper limits at 95\% CL on $\mathscr{B}\left(N^{ \pm} \rightarrow Z l^{ \pm}\right) \mathscr{B}\left(N^{0} \rightarrow W^{ \pm} l^{\mp}\right)$ versus $m_{N}$, assuming $\left|V_{e}\right|=0.055,\left|V_{\mu}\right|=0.063$, and $\left|V_{\tau}\right|=0$. [3] 


\section{Excited Leptons}

In models of composite fermions, the Standard Model quarks and leptons are hypothesized to be bound states of more fundamental constituents. As a consequence of compositeness, new interactions among fermions emerge near the compositeness scale $\Lambda$, including the possibility of excited states of quarks and leptons. This analysis searches for single production of excited leptons through a four-particle contact interaction, $q \bar{q} \rightarrow l l^{*}$, with the excited lepton decaying via $l^{*} \rightarrow l \gamma$. The dataset consists of $13 \mathrm{fb}^{-1}$ of $p p$ collisions at $\sqrt{s}=8 \mathrm{TeV}$ [8].

\subsection{Event Selection}

Events were collected with two triggers: a calorimeter-only trigger requiring two electromagnetic clusters with $p_{T}$ (leading) $>35 \mathrm{GeV}$ and $p_{T}$ (subleading) $>25 \mathrm{GeV}$ for the $e^{*}$ search, and a single-muon trigger requiring $p_{T}>24$ for the $\mu^{*}$ search. The event are required to have two same-flavor, opposite-sign leptons and a photon.

The leptons are required to have transverse momentum 5(1) GeV above the trigger thresholds for electrons (muons), and must additionally pass particle identification, isolation, and impact parameter requirements [8]. The leptons are restricted to the same $\eta$ regions described in section 2.1. The photons are required to have $p_{T}>30 \mathrm{GeV}$, to satisfy ATLAS tight identification requirements [9], and to be isolated. Both unconverted photons, with a calorimeter energy deposition and no inner detector track, and photons that convert to an electron-positron pair are considered. As the model predicts the photon to be well-separated from the leptons, only photons with $\Delta R(\gamma, l)>0.7$ are considered.

\subsection{Backgrounds and Results}

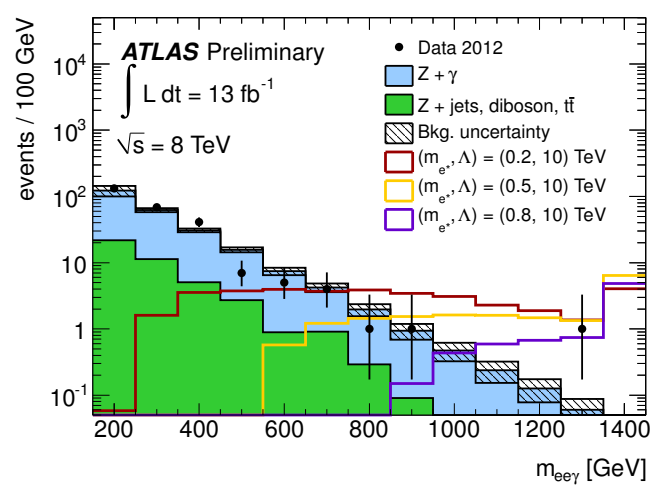

(a)

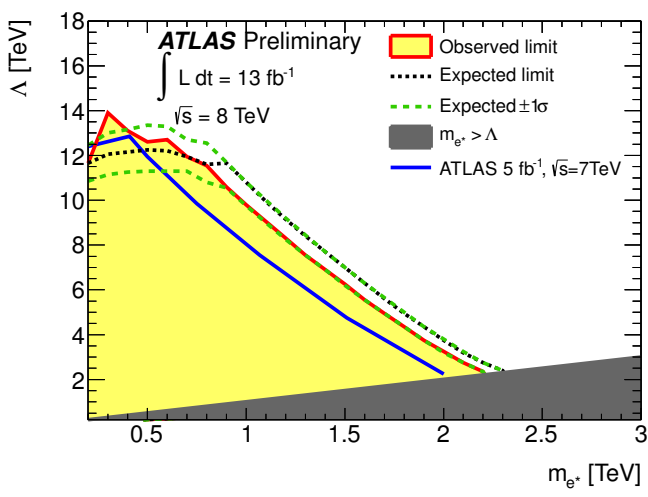

(b)

Figure 2: Left: Distribution of the $l l \gamma$ invariant mass in the signal region. The last bin contains overflow events. Signal predictions for three different $m_{l^{*}}$ hypotheses with $\Lambda=10 \mathrm{TeV}$ are also shown. Right: Exclusion limits in the $m_{l^{*}} \Lambda$ parameter space. Regions to the left of the experimental limits are excluded at 95\% CL. No limits are set in the grey region $m_{l^{*}}>\Lambda$ where the model in not applicable. [8] 
The dominant background to the $l l \gamma$ final states, from Drell-Yan processes, is suppressed by requiring $m_{l l}>110 \mathrm{GeV} . Z+\gamma$ production remains the dominant background after this cut, with smaller contribution from $Z+$ jets where the jet is misidentified as a photon. Both backgrounds are taken from simulation; the rate of jet-to-photon misidentification is found to be overestimated in simulation, so scale factors are derived from the control region with $70 \mathrm{GeV}<m_{l l}<110 \mathrm{GeV}$, and applied to the predicted the $Z+$ jets background. Finally, the Monte Carlo statistics are poor after requiring $m_{l l}>110 \mathrm{GeV}$, so the backgrounds are fitted with an exponential.

The observed and predicted $m_{l l \gamma}$ distribution is shown for the $e^{*}$ case in figure 2a. No signficant excesses are observed, so limits are set on $m_{e^{*}}$ and $m_{\mu^{*}}$, shown for the $e^{*}$ case in figure $2 \mathrm{~b}$.

\section{Model-Independent Trilepton Search}

To provide broad sensitivity to models producing multilepton final states, a model-independent search in trilepton final states was conducted. The analysis uses $4.6 \mathrm{fb}^{-1}$ of $p p$ collisions at $\sqrt{s}=$ $7 \mathrm{TeV}$ [10], and requires three well-identified, energetic, prompt, and isolated leptons, where the first two leptons are electrons or muons, and the third lepton is an electron, muon, or single-prong hadronically decaying tau. Events are separated into four categories, based on whether the third lepton is a hadronically decaying tau, and whether two leptons are consistent with the decay of an on-shell $Z$ boson. Counting experiments are performed in 19 signal regions, shown in table 1, chosen to be sensitive to a range of production and decay modes resulting in multilepton final states.

\begin{tabular}{ccc}
\hline \hline Variable & Lower Bound $(\mathrm{GeV})$ & Add'l Requirement \\
\hline$H_{\mathrm{T}}^{\text {leptons }}$ & $0,100,150,200,300$ & - \\
$E_{\mathrm{T}}^{\text {miss }}$ & $0,50,75$ & $H_{\mathrm{T}}^{\text {jets }}<100 \mathrm{GeV}$ \\
$E_{\mathrm{T}}^{\text {miss }}$ & $0,50,75$ & $H_{\mathrm{T}}^{\text {jets }} \geq 100 \mathrm{GeV}$ \\
$m_{\text {eff }}$ & $0,150,300,500$ & - \\
$m_{\mathrm{eff}}$ & $0,150,300,500$ & $E_{\mathrm{T}}^{\text {miss }}>75 \mathrm{GeV}$ \\
\hline \hline
\end{tabular}

Table 1: Signal regions for the multilepton analysis. Each region is defined by a lower bound on the corresponding variable. $H_{\mathrm{T}}^{\text {leptons }}$ is the scalar sum of $p_{\mathrm{T}}$ of all leptons; $H_{\mathrm{T}}^{\text {jets }}$ is the scalar sum of $p_{\mathrm{T}}$ of all jets; and $m_{\mathrm{eff}}$ is the sum of $H_{\mathrm{T}}^{\text {leptons }}, H_{\mathrm{T}}^{\text {jets }}$, and $E_{\mathrm{T}}^{\text {miss }}$.

\subsection{Backgrounds and Results}

The dominant backgrounds depend on the category and signal region; generally, the backgrounds to the categories with three electrons or muons are primarily from irreducible processes, while the backgrounds to categories with a hadronically decaying tau lepton are largely nonprompt/fake. The irreducible backgrounds consist mostly of the Standard Model $W Z$ and $Z Z$ processes with smaller contributions from $t \bar{t}+W / Z$, and are estimated using simulation. The non-prompt/fake backgrounds are estimated using a data-driven technique that characterizes the properties of such leptons with loosened selection criteria [10].

No significant excesses beyond Standard Model predictions are observed. The results are presented as $95 \%$ CL limits on production of trilepton events for each category and signal region; 


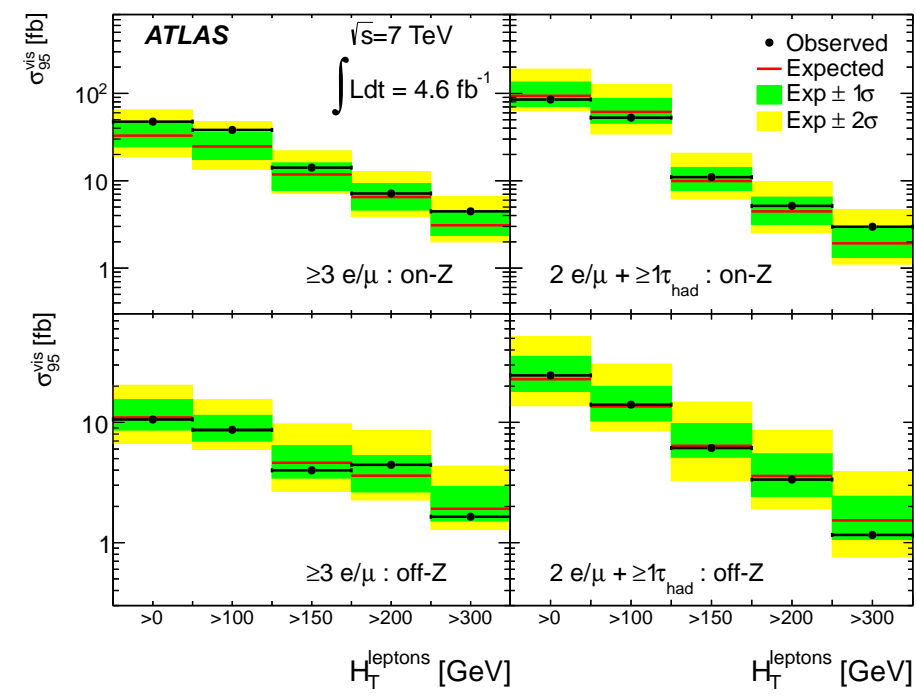

Figure 3: Examples of observed and expected $95 \%$ confidence level upper limits, $\sigma_{95}^{\mathrm{vis}}$, on non-SM trilepton event production, shown for the $H_{\mathrm{T}}^{\text {leptons }}$ signal regions, in the four categories defined by whether the event includes a hadronically decaying tau lepton, and whether the event contains a lepton pair consistent with the decay of an on-shell $Z$ boson. [10]

examples are shown for the $H_{\mathrm{T}}^{\text {leptons }}$ signal regions in figure 3. The limits in all categories and signal regions are available on HEPDATA ${ }^{1}$, along with lepton fiducial efficiencies for model testing.

\section{Conclusion}

Three ATLAS searches for new phenomena in final states with multiple leptons are presented. The searches for type III seesaw heavy fermions and excited leptons utilize model-specific selection criteria to suppress backgrounds, while the model-independent trilepton search emphasizes datadriven techniques to estimate the non-prompt/fake backgrounds accurately. No significant excesses beyond Standard Model background predictions were observed, and limits were set on the models.

\section{References}

[1] ATLAS Collaboration. JINST 3 (2008) S08003.

[2] A. Abada, C. Biggio, F. Bonnet, M. Gavela, and T. Hambye. Phys.Rev. D78 (2008) 033007, arXiv:0803.0481 [hep-ph].

[3] ATLAS Collaboration. ATLAS-CONF-2013-019. http://cds.cern.ch/record/1525526.

[4] ATLAS Collaboration. Eur.Phys.J. C72 (2012) 1909, arXiv:1110.3174 [hep-ex] .

[5] ATLAS Collaboration. Tech. Rep. ATLAS-CONF-2012-125, CERN, Geneva, Aug, 2012.

[6] F. del Aguila, J. de Blas, and M. Perez-Victoria. Phys.Rev. D78 (2008) 013010, arXiv:0803.4008 [hep-ph].

\footnotetext{
${ }^{1}$ http://inspirehep.net/record/1204447/hepdata
} 
[7] C. Biggio and F. Bonnet. Eur.Phys.J. C72 (2012) 1899, arXiv:1107.3463 [hep-ph] .

[8] ATLAS Collaboration. ATLAS-CONF-2012-146. http: / / cds . cern. ch/record/1493485.

[9] ATLAS Collaboration. Phys.Rev. D83 (2011) 052005, arXiv:1012.4389 [hep-ex] .

[10] ATLAS Collaboration. Phys.Rev. D87 (2013) 052002, arXiv:1211.6312 [hep-ex] . 